\title{
Development of a Combined Standard Additions/Internal Standards Method to Quantify Residual PEG in Ethoxylated Surfactants by MALDI TOFMS
}

\author{
Renata Szyszka, ${ }^{1}$ Scott D. Hanton, ${ }^{2,3}$ Diane Henning, ${ }^{2}$ Kevin G. Owens ${ }^{1}$ \\ ${ }^{1}$ Department of Chemistry, Drexel University, 3141 Chestnut Street, Philadelphia, PA 19104, USA \\ ${ }^{2}$ Air Products and Chemicals, Inc., Allentown, PA USA \\ ${ }^{3}$ Intertek, Inc., 7201 Hamilton Blvd, RD1, Allentown, PA 18195, USA
}

\begin{abstract}
Measuring the residual polyethylene glycol (PEG) in polyethylene oxide (PEO)-based surfactants is important to fully understanding the performance of these materials. Traditional methods of quantitating PEG in PEO-based surfactants can be time-consuming and struggle with low amounts or overlapping molecular mass distributions. This paper describes a matrixassisted laser desorption/ionization (MALDI) mass spectrometry method developed to quantitate residual PEG in a series of ethoxylated surfactants. The technique addresses the difficulties faced in doing quantitative MALDI experiments by utilizing both internal standard and standard additions protocols. The method produces excellent straight line standard addition plots, and the quantitative results are verified using both a constructed standard and an independent traditional chromatographic separation.
\end{abstract}

Key words: Polymer quantitation, Quantitative MALDI TOFMS, PEG analysis, PEG contamination, Ethoxylated surfactant

\section{Introduction}

$\mathrm{C}$ ommercially available synthetic poly(ethylene oxide) (PEO)-based nonionic surfactants are widely used for various applications [1]. These polymers are typically the products of a condensation reaction between ethylene oxide and a molecule with an available $-\mathrm{OH}$ function. The simplest PEO surfactant is the result of reaction with water. More complicated PEO-based surfactants with the formula $\mathrm{R}_{1}\left(\mathrm{OCH}_{2} \mathrm{CH}_{2}\right)_{\mathrm{n}} \mathrm{OR}_{2}$, where $\mathrm{R}_{1}$ and $\mathrm{R}_{2}$ are generally hydrophobic end groups, are the products of the reaction of ethylene oxide and different alcohols. These non-ionic surfac-

\footnotetext{
Electronic supplementary material The online version of this article (doi:10.1007/s13361-010-0065-9) contains supplementary material, which is available to authorized users.
}

Correspondence to: Kevin G. Owens; e-mail: kevin.owens@drexel.edu tants are characterized by their exceptional dispersing properties, wetting, emulsification, and detergency behaviors [2]. They are nontoxic materials that are widely used to reduce surface tension, regulate viscosity [3], and act as stabilizers [4].

In the production of PEO-based surfactants, it is often important to limit the amount of water present in the reactor. Any residual water will also be ethoxylated to create polyethylene glycol (PEG). Since a mixture of two surfactants often creates a surfactant formulation that exhibits different physical properties than either of the individual components, control of the amount of PEG in an ethoxylated surfactant is important.

Matrix-assisted laser desorption/ionization (MALDI) mass spectrometry has been shown to be an effective analysis technique to characterize polymer materials [511]. MALDI methods have been developed to analyze a wide range of polymer chemistry, especially materials like the 
majority of commercial PEOs. Typical PEO-based surfactant materials are relatively low molar mass, are relatively hydrophilic, and exhibit a relatively narrow polydispersity. All of these characteristics make these materials very suitable for MALDI analysis. The MALDI mass spectra can provide detailed chemical structure information about the PEO surfactants. The ethylene oxide repeat units are readily demonstrated, and mass information about the end groups can be determined. The average molar masses of the polymer materials can also be readily determined.

While MALDI has been very effective at measuring the chemical structure of individual polymer materials, it has generally struggled to effectively quantitate mixtures of different polymers [12]. MALDI data can be used to identify different components in a blend through repeat unit and end group identification and measurement of average molar masses, but quantifying the relative amounts of the components in the blend is difficult. The challenges in using MALDI to effectively quantitate polymer blends have both molar mass [13] and chemical function (repeat unit and end group) causes [12].

Despite these challenges, there have been a few reports of successful application of MALDI to quantitative problems in biotechnology, such as low mass peptides (i.e., $<500 \mathrm{Da}$ ) [14-16], larger proteins [17, 18], food oligosaccharides [19], anthocyanins in red wine [20], spirolide toxins in phytoplankton samples [21], ribonucleic acids (RNAs), and post-transcriptionally modified nucleosides [22]. There have been a few isolated reports of successful quantitation in problems involving synthetic polymers. Wood and co-workers demonstrated that quantitative measurements of polydimethylsiloxane (PDMS) type polymers could be performed using the relative ratio of the signal intensities of two molar mass distributions of the same PDMS [23]. Chen and He went one step farther and investigated the use of an internal standard (IS) for the quantitative determination of three polymers with different end groups using poly(ethylene)glycol methyl ether (PEG-OMe) as the IS [24].

Conventionally, polymer blends are typically analyzed using liquid chromatography techniques such as high performance liquid chromatography (HPLC) or size exclusion chromatography (SEC), or spectroscopy techniques such as nuclear magnetic resonance (NMR) or infrared absorption (IR). All of these techniques can be powerful tools to measure polymer blends; however, the spectroscopic tools have difficulty distinguishing blends of different polymers with the same repeat units, especially if the end groups are either similar, or if the desired component is of relatively low concentration. SEC is a powerful technique to analyze blends of polymers that have different molar masses, but cannot separate polymer blends with greatly overlapping molar masses. To this point, HPLC has been the technique of choice to analyze chemically similar polymer blends. While HPLC has been shown to solve these types of problems, the methods can be difficult to develop and time-consuming to run.
The goal of this work is to demonstrate a MALDI-based method to quantitate chemically similar polymer blends. The internal standard based method of Chen and He [24] is extended to include standard additions (SA) protocols [25]. The combination of the IS and SA sample preparation helps compensate for the variation in absolute signal intensities from the MALDI experiment. These variations are poorly understood and are due to many different variables, including sample preparation method, sample deposition method, laser fluence, instrument conditions, etc.

In these studies of polymer blends, the PEO-based surfactant serves as the internal standard and the measured signal of the PEG contaminant is normalized to the signal of the surfactant. As customary in any SA experiment, for each analyte material, a series of analytical samples is created containing the same amount of analyte and increasing quantities of a PEG standard. The selected group of compounds for this research was non-ionic ethoxylated surfactants with different $R_{1}$ and $R_{2}$ end groups.

\section{Experimental}

\section{Materials}

Five commercial PEG polymers, with average molar masses of 400,1000,1500, 2000, and $3500 \mathrm{Da}$ obtained from Aldrich Chemical Company (Milwaukee, WI, USA) were used as standards for the standard additions curves. Ethoxylated surfactants were obtained from several different sources. The Igepal Co890, Brij 98, and poly(ethylene glycol) methylether 2000, denoted PEG-OMe (A) were from Aldrich. Ethoxylated octadecanol and Triton X305 were purchased from BASF Corporation (Monaca, PA, USA). Brij 35 was purchased from Pierce Chemical Company (Rockford, IL, USA, now a part of Thermo Fisher). A second poly(ethylene glycol) methylether 2000 sample, denoted PEG-OMe (PS), was purchased from Polymer Source Inc. (Dorval, Montreal, Canada). An experimental ethoxylated surfactant denoted D-50 was supplied by Air Products and Chemicals, Inc. (Allentown, PA, USA).

Three MALDI matrices were used in these experiments: 2,5-dihydroxybenzoic acid (DHB), $\alpha$-cyano-4-hydroxycinnamic acid (CHCA), and trans-3-indoleacrylic acid (IAA). All were purchased from Aldrich. The DHB was re-crystallized from water purified using a Barnstead E-Pure system before use. Sodium trifluoroacetate (NaTFA) from Aldrich was used as the cationization reagent. All polymers, matrices, and cationization reagent were dissolved in tetrahydrofuran (THF) purchased from Aldrich.

\section{Sample Preparation}

Working solutions of the matrix were prepared by dissolving the solid material in THF to a concentration of $0.25 \mathrm{M}$. A working solution of the cationization agent (also termed the salt) was prepared by dissolving NaTFA in THF to a final 
concentration of $2.5 \mathrm{mM}$. PEG standards were made by dissolving the polymer in THF to obtain $0.1 \mathrm{mg} / \mathrm{mL}$ working solutions. Ethoxylated surfactant analyte solutions were all made to a concentration of $5 \mathrm{mg} / \mathrm{mL}$ of polymer in THF. Samples for MALDI analysis were prepared by mixing the chosen surfactant analyte, PEG standard, MALDI matrix, and NaTFA salt solutions at a selected matrix-to-analyte (M/ A) and salt-to-analyte (S/A) ratio. Table $\mathrm{S} 1$ (in the Supplemental Information) shows the detailed sample preparation scheme for a single standard additions experiment. For these experiments, the M/A ratio was chosen to range from 2000 to 3000 . The S/A ratio was kept at a constant value of 2:1 [26].

The sample solutions were deposited on the MALDI target plate either using the dry-drop method or the electrospray deposition method [27]. For the dry-drop method, $0.5 \mu \mathrm{L}$ aliquots were deposited on the sample plate and allowed to dry under ambient conditions. For electrospray deposition, we followed the protocol developed by Erb and co-workers: the distance from the needle to the sample plate was set to $2 \mathrm{~cm}$, the potential applied to the stainless steel needle was approximately $5.8 \mathrm{kV}$, the flow rate was set to $5 \mu \mathrm{L} / \mathrm{min}$ and the spray time was $5 \mathrm{~s}$ [28].

\section{Instrumentation}

All mass spectra were acquired on a Bruker (Bremen, Germany) Reflex III MALDI TOFMS instrument. The analyses were performed in positive ion reflectron mode using a medium pulsed ion extraction (PIE) delay time. The instrument parameters were optimized for best mass resolution at $2000 \mathrm{Da}$. All the mass spectra were recorded at a $1 \mathrm{Gs} / \mathrm{s}$ data sampling rate. The laser fluence was selected to be just above threshold for the observation of MALDI ions. Each individual mass spectrum was the sum of 50 shots acquired from the whole sample by rastering the laser spot across the sample surface. Data was acquired using the Bruker XACQ ver. 4.0 software running on a Sun (Sunnyvale, CA, USA) SPARCstation 5 workstation. An average of four mass spectra was obtained from every sample spot and at every concentration level.

\section{MS Data Analysis}

Mass spectral data processing was performed using Polymerix ver. 2.0 from Sierra Analytics, LLC (Modesto, CA, USA). The total peak area of all oligomers of the PEG contaminant signal, i.e., the sum of PEG peak areas $\left(A_{P E G}\right)$ was normalized to the total peak area of all oligomers of the analyte internal standard, $\left(A_{I S}\right)$. The concentration of residual PEG in the unknown is conceptually determined in the normal standard additions fashion by extending the curve fitted to the plot of peak area ratio $\left(A_{P E G} / A_{I S}\right)$ versus wt\% $\mathrm{PEG}$ added to its intersection with the $x$-axis; practicaly, the values are calculated by dividing the value of the $y$-intercept by the slope of the fitted regression equation. The relative standard deviation (RSD) of peak area ratios determined from replicate mass spectral measurements was calculated to compare the reproducibility of analyte and internal standard ion yields. All statistical analyses were carried out using Microsoft Excel 2003.

\section{HPLC}

HPLC analysis of the experimental D-50 material was performed on an Agilent (Wilmington, DE, USA) model 1100 high-performance liquid chromatograph employing an Alltech (Deerfield, IL, USA) evaporative light scattering detector (ELSD). The $5 \mathrm{mg} / \mathrm{mL}$ analyte sample described above was diluted 1:10 in methanol (Aldrich) and $100 \mu \mathrm{L}$ was injected. The sample was analyzed in triplicate. The D-50 sample was separated on an Alltech Platinum EPS C18 column $(3 \mu \mathrm{m}$ particle size, $150 \mathrm{~mm}$ long $\times 2.1 \mathrm{~mm}$ diameter column) run at $40{ }^{\circ} \mathrm{C}$. A reverse phase water:methanol gradient ran from $65 \%$ water to $5 \%$ water. The ELSD was operated with the impactor on, at $40{ }^{\circ} \mathrm{C}$, and with $1.5 \mathrm{~L} /$ $\min \mathrm{N}_{2}$ flow.

\section{Results and Discussion}

\section{Method of Standard Additions}

In this work, the ion signal from the added PEG standard and the ethoxylated surfactant analyte are observed simultaneously. Each standard additions experiment requires the analysis of seven binary mixtures with different amounts of the PEG standard added to the IS analyte. The relative signal intensity of the PEG peaks is found to increase linearly with the amount of PEG standard added to the sample. This is demonstrated in Figure S1 (in the Supplemental Information) where the growth of the relative signal intensity of the added PEG standard (denoted as A) to the PEG-OMe (PS) internal standard (denoted as $\mathrm{C}$ ) is shown. Figure 1 shows a typical MALDI mass spectrum from the standard additions experiment involving the addition of $50 \mathrm{wt} \%$ PEG 1500 to the PEG-OMe (PS) analyte. The lower molecular mass distribution (marked as A) in Figure 1a is of the added PEG standard, while the higher molecular mass distribution (marked C) is of the ethoxylated surfactant. Note that as shown in the expansion in Figure 1b, the peaks in the isotope clusters are clearly resolved; however, there is overlap in signal from oligomers of the different components of the sample. In Figure 1b the isotope cluster for the sodium cationized PEG-OMe oligomer at $\mathrm{m} / z$ 1728.01 Da overlaps with the isotope cluster of the potassium cationized PEG oligomer at $m / z 1729.97 \mathrm{Da}$ (in each case the monoisotopic mass corresponding to oligomers with a degree of polymerization (DP) of 38). While the sodium and potassium cationized oligomers were present in most of the spectra measured, the sodium-adduct peaks were generally used for quantitation due to their greater intensity. In the case of an overlap such as this, only the first two peaks in the PEG$\mathrm{OMe}$ isotope cluster were integrated and used in the standard 


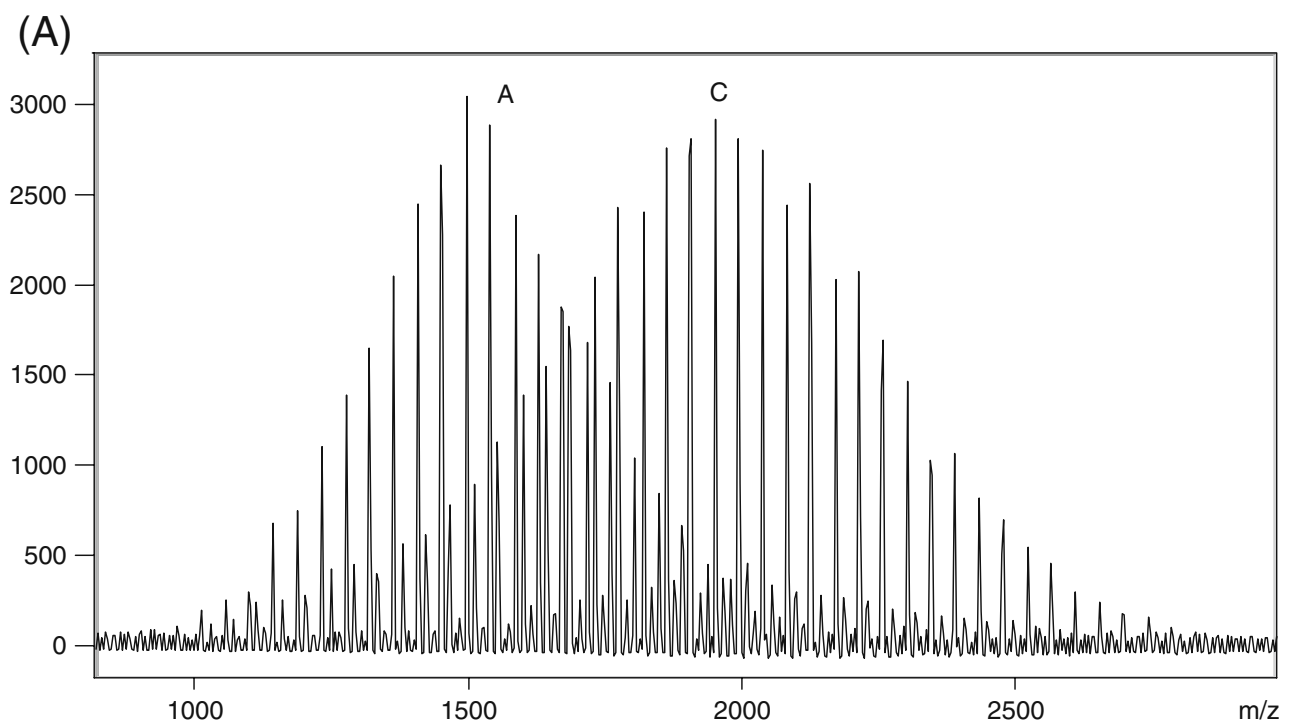

(B)

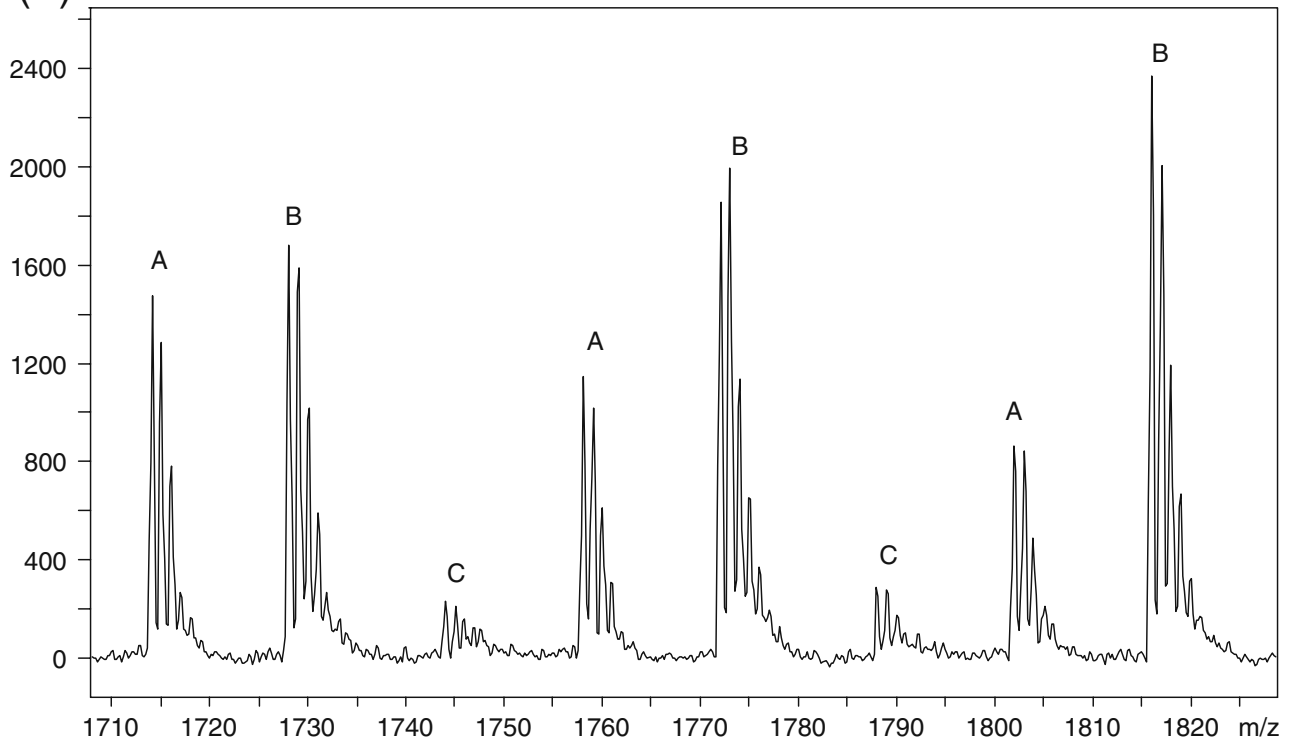

Figure 1. MALDI TOFMS spectrum of a mixture of 50 wt\% PEG and PEG-OMe (PS). The upper trace (A) shows two full polymer distributions The expansion in trace (B) shows the three isotope clusters observed: $A[P E G+N a]^{+}, B$ an overlap of $[\mathrm{PEG}-\mathrm{OMe}+\mathrm{Na}]^{+}$and $[\mathrm{PEG}+\mathrm{K}]^{+}$, and $\mathrm{C}[\mathrm{PEG}-\mathrm{OMe}+\mathrm{K}]^{+}$

additions calculations. In all cases studied there was sufficient separation of any overlapping differently cationized isotope clusters to enable the accurate integration of at least one peak that could be used for the analysis.

Table 1 summarizes the ratios of the measured peak areas of PEG relative to PEG-OMe $\left(A_{P E G} / A_{I S}\right)$ at the seven different PEG concentration levels studied. It also provides relative standard deviations (RSD) and 95\% confidence intervals used to determine the reproducibility of the analysis. The small variability in the signal intensities was obtained due to the use of the ethoxylated surfactant as an IS in the standard additions experiment. Note that the PEG-OMe (PS) sample used in this experiment was spiked with $5.6 \mathrm{wt} \%$ of a PEG 1500 standard, as preliminary work indicated this sample contained an undetectable amount of residual PEG. A plot of the data in
Table 1. Measured peak area ratios and spectrum-to-spectrum variability calculated for the analysis of the PEG-OMe (PS) sample spiked with $5.6 \mathrm{wt} \%$ of a PEG 1500 standard. The number of spectra contributing to the relative standard deviation is 4 , and the coefficient of determination $\left(\mathrm{r}^{2}\right)$ obtained for the analysis is 0.9939

\begin{tabular}{lcccc}
\hline $\begin{array}{c}\text { Concentration } \\
\text { of added PEG } \\
\text { relative to } \\
\text { PEG-OMe [wt } \%]\end{array}$ & $\begin{array}{c}\text { Absolute } \\
\text { concentration } \\
\text { of PEG added } \\
{[\mu \mathrm{M}]}\end{array}$ & $\mathrm{A}_{\mathrm{PEG}} / \mathrm{A}_{\text {IS }}$ & $\begin{array}{c}\text { Relative } \\
\text { SD [\%] }\end{array}$ & C.I. \\
\hline 0.0 & 0 & 0.07 & 4.6 & 0.005 \\
0.75 & 0.9 & 0.07 & 6.8 & 0.008 \\
2.27 & 2.6 & 0.09 & 5.9 & 0.009 \\
3.80 & 4.3 & 0.10 & 2.3 & 0.004 \\
7.70 & 7.98 & 0.11 & 4.4 & 0.008 \\
20.0 & 16.8 & 0.26 & 1.8 & 0.008 \\
42.9 & 26.8 & 0.51 & 4.3 & 0.035 \\
Average relative SD & & & 4.31 & \\
\hline
\end{tabular}


Table 1 yields a linear relationship $(y=0.0104 x+0.0583)$ with a coefficient of determination $\left(\mathrm{r}^{2}\right)>0.99$. To complete the standard additions calculation to determine the amount of PEG present in the spiked PEG-OMe (PS) sample, the value of the $\mathrm{y}$-intercept is divided by that of the slope to calculate the $\mathrm{wt} \%$ of PEG in the unknown. For this sample the amount of PEG contamination was determined to be $5.6( \pm 0.1) \mathrm{wt} \%$, in excellent agreement with expectation.

\section{Effect of Surfactant End Group on Internal Standard Response}

With the excellent results obtained for the determination of the quantity of PEG contaminant in the spiked PEG-OMe (PS) sample, we extend this study to determine the amount of PEG contamination in a series of ethoxylated surfactants with different end groups. The surfactants in the set were chosen to have similar average molar masses. Previous work has shown the difficulties in making quantitative polymer blend measurements on polymers with significantly different average molecular masses. As shown in Table 2, the chemical structure of the end groups of the ethoxylated surfactants varied significantly.

Figure 2 shows the plots of the PEG standard signal response normalized to the ethoxylated surfactant signal from the standard additions experiments. As observed for the first case, the relationships are remarkably linear for every polymer investigated. Table 3 contains the results of the standard additions experiments, including the slope, intercept, $\mathrm{r}^{2}$, and the calculated amount of residual PEG in each ethoxylated surfactant.

These experiments have produced very consistent straight line results with every experiment having an $r^{2}$ value $>0.97$, and most being $>0.99$. These ethoxylated surfactants show a wide range of residual PEG homopolymer in the final material. Note that one of the PEG-OMe samples in the study [i.e., PEG-OMe (PS)] has no detected residual PEG, while the PEG-OMe ${ }_{2}$ sample contains approximately $21 \mathrm{wt} \%$ PEG contaminant.

In both Figure 2 and Table 3, we observe significantly different apparent responses (i.e., slopes of the standard addition plots) for the various ethoxylated surfactants. As the slope is the ratio of the measured PEG signal to that of the ethoxylated surfactant IS, this suggests that the response

Table 2. End groups of the ethoxylated surfactants used in the standard additions study

\begin{tabular}{ll}
\hline Ethoxylated surfactant & \multicolumn{1}{c}{ End groups } \\
\hline PEG-OMe (PS) & $\mathrm{CH}_{3} \mathrm{OH}$ \\
PEG-OMe (A) & $\mathrm{CH}_{3} \mathrm{OH}$ \\
Brij 35 & $\mathrm{C}_{12} \mathrm{H}_{25} \mathrm{OH}$ \\
D-50 & $\mathrm{C}_{10}-\mathrm{C}_{14}$ alcohols \\
Octadecanol + EO & $\mathrm{C}_{18}$ alcohol \\
Brij 98 & $\mathrm{C}_{18} \mathrm{H}_{35} \mathrm{OH}$ \\
Igepal Co 890 & $\mathrm{C}_{8}$-phenol \\
Triton X305 & $\mathrm{C}_{9}$-phenol \\
PEG-OMe2 & $\mathrm{CH}_{3} \mathrm{OCH} \mathrm{H}_{3}$ \\
\hline
\end{tabular}

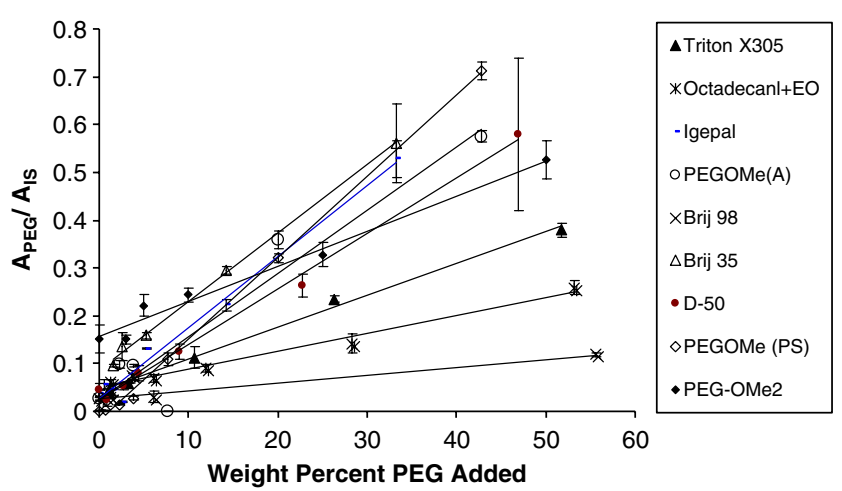

Figure 2. Standard additions curves obtained for the eight different surfactants investigated. The plot is of the average peak area ratios for PEG to internal standard plotted against wt $\%$ of added PEG standard. Error bars represent the 95\% confidence intervals

depends in some way on the contributions of the different end groups for the analytes. The apparent response in the MALDI experiment of the different polymer materials does not appear to correlate with the relative hydrophilicity or hydrophobicity of the end group. The response also does not appear to correlate with the relative strength of the surfactant as defined by the hydrophilic-lipophilic balance (HLB) value. The different responses observed in these experiments is interesting and, with further work, may provide a better understanding of the different responses of polymer materials observed in the MALDI experiment.

\section{Effect of Choice of the MALDI Matrix}

All of the standard addition experiments described above were completed using DHB as the MALDI matrix. To explore the influence of the choice of matrix on the standard additions results, the PEG-OMe (PS) sample was spiked with $5.4 \mathrm{wt} \%$ of the PEG1500 standard and then prepared as described in the 'Experimental section' with three different matrices. Except for the laser energy, all other instrumental parameters were kept constant. Note that the laser energy had to be optimized for each matrix due to the very different molar absorptivities of these materials at the nitrogen laser wavelength. As shown in Figure 3, the measurements generated straight lines characterized by high coefficients of determination $\left(\mathrm{r}^{2}>0.99\right)$. Table 4 provides a summary of the data obtained in the regression analysis.

This set of experiments shows a small effect of the choice of the MALDI matrix. There are small but statistically significant differences in the quantity of the residual PEG determined using the three different MALDI matrices. While these differences are statistically significant, differences this small are generally not significant to the polymer and formulation chemists, as the $0.2 \mathrm{wt} \%$ differences observed will have little impact on the surfactant properties of the mixture. 
Table 3. The determined quantity of PEG contaminant in the ethoxylated surfactants studied using the combined internal standard additions MALDI method

\begin{tabular}{lcccc}
\hline \multicolumn{1}{c}{ Surfactant } & Slope & Intercept & $\mathrm{r}^{2}$ & wt \% PEG \\
\hline PEG-OMe (PS) & 0.0145 & -0.0247 & 0.996 & ND \\
PEG-OMe (A) & 0.0129 & 0.0494 & 0.981 & $3.8( \pm 0.1)$ \\
Brij 35 & 0.0143 & 0.0863 & 0.990 & $0.077^{\mathrm{a}}$ \\
D-50 & 0.0115 & 0.0236 & 0.989 & $2.1( \pm 0.3)$ \\
Octadecanol + EO & 0.0033 & 0.0326 & 0.990 & $9.8( \pm 0.1)$ \\
Brij 98 & 0.0017 & 0.0249 & 0.997 & $0.3( \pm 0.8)$ \\
Igepal Co 890 & 0.0169 & 0.0046 & 0.993 & $6.4( \pm 0.1)$ \\
Triton X305 & 0.0067 & 0.0426 & 0.980 & $21.0( \pm 0.1)$ \\
PEG-OMe & 0.0074 & 0.155 & & \\
\hline
\end{tabular}

$N D$ not detected

${ }^{a}$ Potassium cationized peaks included in total peak area of both polymers

\section{Effect of Molar Mass of the Selected PEG Standard}

In previous work, we have observed important issues with quantitative measurements of polymer blends when the polymers composing the blend have significantly different average molar masses [13]. To extend our understanding of the role of the polymer average molar mass in these quantitation studies, we investigated the influence of the average molar mass of the PEG standard used in the standard addition experiments. We examined adding PEG standards having average molar masses of 1000, 1500, 2000, and $3500 \mathrm{Da}$ to the PEG-OMe (PS) sample, which was spiked with $5.4 \mathrm{wt} \%$ of the PEG1500 standard. Figure S2 in the Supplemental Information shows representative mass spectra from these experiments.

The results in Table 5 show a significant influence of the average molar mass of the added PEG standard on the measured $\mathrm{wt} \%$ PEG determined from the standard additions experiments. These results verify previous observations that average molecular mass plays a significant role in the ability to accurately quantify polymer blends [29-31]. Our interpretation of these results is that the most accurate $\mathrm{wt} \%$ PEG quantitation will be obtained when the added PEG is closest in average molar mass to the observed residual PEG in the ethoxylated surfactant.

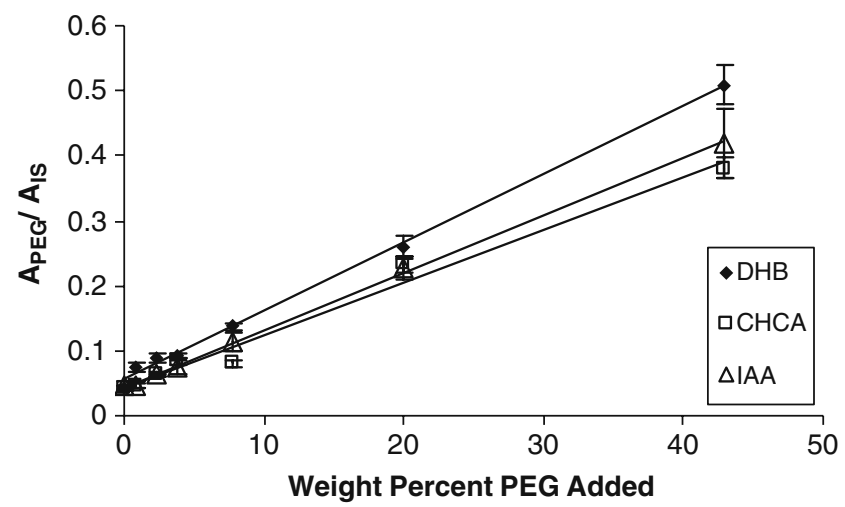

Figure 3. Standard additions curves for determination of the wt\% PEG in the PEG-OMe (PS) sample spiked with $5.4 \mathrm{wt} \%$ PEG 1500 in three MALDI matrices: DHB, CHCA, and IAA. Error bars represent the $95 \%$ confidence intervals
It is interesting to note that the observed differences with average molar mass are not randomly distributed. We observe a nominally linear change in the difference between the calculated and expected quantity of PEG with an increase in the average molecular mass of the added PEG. When a lower average molar mass standard is used (1000 Da), we measure less residual PEG, and as higher molar mass standards are used, we measure increasing amounts of residual PEG in the surfactant analyte. These results may also help us better understand the relative responses of different polymeric materials in the MALDI experiment.

\section{Effect of Choice of Sample Deposition Technique}

The standard additions results given above in Table 3 were obtained from experiments performed using the electrospray deposition technique. Dry-drop deposition was also evaluated to uncover any differences associated with the sample preparation procedure. Dry-drop samples were analyzed in two ways. One set of experiments was performed on samples deposited "all-at-once" on the sample holder. In these experiments, the sample plate with multiple deposited spots was placed in the instrument and mass spectra were acquired from the spots in order from the lowest concentration of PEG added to the highest. A second set of experiments was done on samples deposited using the drydrop technique in a "one-at-a-time" manner, where each sample solution was spotted on the plate and mass analyzed individually. As the matrix/analyte crystals formed by evaporation of the highly volatile THF solvent are extremely small, this "one-at-a-time" technique was used because it was noted that the samples prepared using the "all-at-once" approach visually appeared to evaporate during the relatively

Table 4. Summary of the standard additions data obtained from analysis of the PEG-OMe (PS) sample spiked with $5.4 \mathrm{wt} \%$ PEG1500 using different matrices

\begin{tabular}{lccc}
\hline Matrix & Slope & Intercept & PEG from the slope, $[\mathrm{wt} \%]$ \\
\hline 2,5-DHB & 0.01050 & 0.0549 & $5.2( \pm 0.05)$ \\
CHCA & 0.00808 & 0.0441 & $5.4( \pm 0.09)$ \\
IAA & 0.00884 & 0.0439 & $5.0( \pm 0.07)$ \\
\hline
\end{tabular}


Table 5. Influence of the molar mass of the PEG standard added on the calculated $\mathrm{wt} \%$ PEG in the PEG-OMe (PS) sample spiked with $5.4 \mathrm{wt} \%$ PEG1500

\begin{tabular}{cccc}
\hline $\begin{array}{c}\text { PEG molecular } \\
\text { weight }\end{array}$ & $\begin{array}{c}\text { \%PEG } \\
\text { calculated }\end{array}$ & $\begin{array}{c}\text { Difference } \\
{[\%]^{\mathrm{a}}}\end{array}$ & $\begin{array}{c}\text { Average } \\
\text { \% CV }\end{array}$ \\
\hline 1000 & 1.7 & -69.4 & 6.4 \\
1500 & 5.5 & 1.2 & 3.5 \\
2000 & 13.2 & 142.1 & 3.5 \\
3500 & 43.9 & 705.5 & 4.2 \\
\hline
\end{tabular}

${ }^{a}$ Difference is calculated from spiked value

${ }^{\mathrm{b}}$ Average $\% \mathrm{CV}$ includes data from five replicates

short measurement time. All three experiments generated standard addition plots with high coefficients of determination $\left(r^{2}>0.995\right)$. There was no statistically significant difference between the slopes obtained for the electrospray and "one-at-a-time" dry-drop deposition techniques. There was, however, a statistically significant difference in the slope of the curve obtained for the dry-drop sample preparation when all spots were analyzed "all-at once." For a spiked PEG-OMe (PS) sample with 5.4 wt\% PEG added, the dry-drop "all-at once," dry-drop "one-at-a-time," and electrospray deposition methods yielded results 5.3, 5.2 and $5.5 \mathrm{wt} \%$ PEG, respectively (which correspond to relative errors of $-2.5 \%,-4.8 \%$, and $1.2 \%$ ). Note that these values are all identical within experimental error. While there is a statistically different slope for the "all-at-once" samples, there is apparently a compensating change in the y-intercept value. It may only be fortuitous that the calculated residual PEG values are the same; if the samples were measured in reverse order of concentration, or in a random order of concentration, or if they were prepared at a different matrixto-analyte ratio, the calculated quantity of PEG for the "allat-once" measurements may have been different.

Note that in this case the "one-at-a-time" dry-drop results do agree with those obtained via electrospray deposition. Some may suggest that this argues that electrospray deposition is generally not required in order to obtain highly reproducible results. However, the use of DHB (or CHCA) as a matrix for these ethoxylated materials, particularly with the fast evaporating THF solvent, yields a homogeneous mixture of matrix, analyte, and cationization reagent on the sample surface. It is the homogeneity of the solid MALDI samples that is critical to the highly reproducible MALDI signals observed. In our experience, electrospray deposition is needed when there are larger differences in molecular properties between the different components of the MALDI sample.

\section{Verification of the Standard Additions Method}

The results of the MALDI analysis were first verified using surfactant samples spiked with known amounts of a PEG standard. Since the PEG-OMe (PS) sample was found to contain no detected residual PEG, it was relatively straightforward to construct a sample containing a known amount
(5.6 wt\%) of PEG. This spiked sample was analyzed using the combined standard additions internal standard MALDI method, and returned values of 5.6 and $5.7 \mathrm{wt} \%$, respectively, using the DHB and CHCA matrices. The accuracy of the method (a relative error of 0 and $2 \%$ was determined using the DHB and CHCA matrices) was found to be excellent.

To further verify the MALDI results, they were compared with a standard reversed-phase HPLC-based method using evaporative light scattering detection (ELSD) developed to measure the residual PEG in an ethoxylated surfactant. A sample of the experimental surfactant D-50 was analyzed with the HPLC method, using a PEG 1000 standard to create the calibration curve for the HPLC analysis. Examples of the HPLC chromatograms are shown in the Supplemental Information as Figure S3. The standard additions curve from the MALDI analysis (slope $=0.0115$, intercept $=0.0237$, $\left.\mathrm{r}^{2}=0.9769\right)$ yield a value of $2.1( \pm 0.3) \mathrm{wt} \%$ PEG present, whereas the HPLC results suggest a contamination level of $2.4 \mathrm{wt} \%$ PEG. The MALDI results exhibit a relative error of $-10 \%$, in good agreement with the HPLC based method. Comparable amounts of residual PEG are determined from both techniques, demonstrating that the combined internal standard, standard additions MALDI technique is applicable for quantitative analysis of the residual PEG in these commercially available ethoxylated surfactants.

\section{Conclusions}

This study demonstrates that a combined standard additions and internal standard approach enables MALDI to provide useful quantitative results to measure residual PEG in ethoxylated surfactants. These standard addition experiments provided highly reproducible data with high linearity in the standard addition plots. The standard addition method was verified using spiked standards and by comparison with a traditional HPLC method on one of the analyte samples.

The combination of the standard additions and internal standard protocols has enabled us to address the relative response issues that have plagued previous quantitative MALDI experiments. By building on previous understanding of key aspects of the MALDI experiment, we have controlled important variables such as the sample deposition, the matrix-to-analyte ratio, and the salt-to-analyte ratio. Without an understanding of the impact of these variables on the MALDI experiment, true quantitation would not be possible.

\section{Acknowledgments}

The authors thank Air Products and Chemicals, Inc. for their support of this research, Brian Smith and Mike Williams for the D-50 experimental ethoxylated surfactant, and Dr. Sherri Bassner and Ingrid Meier for critical review of the manuscript. They also thank April Holcomb for help with the 
MALDI instrument and Scott Campbell at Sierra Analytics, LLC, for assistance with the Polymerix analysis.

\section{References}

1. Zabaleta, V., Campanero, M.A., Irache, J.M.: An HPLC with evaporative light scattering detection method for the quantification of PEGs and Gantrez in PEGylated nanoparticles. J. Pharmaceut. Biomed. Anal. 44(5), 1072-1078 (2007)

2. Kwon, T.H., Lee, E.S., Lee, S.M., Bae, J.Y.: Synthesis of TRITON Xbased phosphate ester surfactants and their self-charring behavior. Polym. Degrad. Stab. 92, 1546-1554 (2007)

3. Kataoka, K., Harada, A., Nagasaki, Y.: Block copolymer micelles for drug delivery: design, characterization and biological significance. $A d v$. Drug Deliv. Rev 47, 113-131 (2001)

4. Ould-Ouali, L., Arien, A., Rosenblatt, J., Nathan, A., Twaddle, P., Matalenas, T., Borgia, M., Arnold, S., Leroy, D., Dinguizli, M., Rouxhet, L., Brewster, M., Preat, V.: Biodegradable self-assembling PEG-copolymer as vehicle for poorly water-soluble drugs. Pharmaceut. Res. 21(9), 1581-1590 (2004)

5. Hanton, S.D.: Mass spectrometry of polymers and polymer surfaces. Chem. Rev. 101(2), 527 (2001)

6. Nielen, M.W.F.: MALDI time-of-flight mass spectrometry of synthetic polymers. Mass Spectrom. Rev. 18, 309 (1999)

7. Montaudo, G., Lattimer, R.P. (eds.): Mass Spectrometry of Polymers. CRC Press, Boca Raton (2002)

8. Pasch, H., Schrepp, W. (eds.): MALDI-TOF Mass Spectrometry of Synthetic Polymers. Springer-Verlag, Berlin (2003)

9. Wallace, W.E., Guttman, C.M., Hanton, S.D.: Quantitative synthetic polymer mass spectrometry workshop. J. Res. Natl. Inst. Stand. Technol. 108, 79-85 (2003)

10. Wallace, W.E., Guttman, C.M., Wetzel, S.J., Hanton, S.D.: Mass spectrometry of synthetic-polymer mixtures workshop. Rapid Commun. Mass Spectrom. 18, 518-521 (2004)

11. Li, L. (ed.): MALDI Mass Spectrometry for Synthetic Polymer Analysis. Wiley, Hoboken (2010)

12. Hanton, S.D., Owens, K.G.: MALDI MS for Industrial Polymers in Li, L. (ed). MALDI Mass Spectrometry for Synthetic Polymer Analysis, John Wiley \& Sons: Hoboken, NJ (2010).

13. Goldschmidt, R.J.: Some quantitative aspects of the analysis of synthetic polymers by MALDI TOFMS, Ph.D. Thesis, Drexel University, 1998.

14. Bucknall, M., Fung, K.Y.C., Duncan, M.W.: Practical quantitative biomedical applications of MALDI-TOF mass spectrometry. J. Am. Soc. Mass Spectrom. 13, 1015-1027 (2002)

15. Duncan, M.W., Matanovic, G., Cerpa-Pojak, A.: Quantitative analysis of low molecular weight compounds of biological interest by matrixassisted laser desorption ionization. Rapid Commun. Mass Spectrom. 7, 1090-1094 (1993)

16. Sparbier, K., Wenzel, T., Dihazi, H., Blaschke, S., Mueller, G.A., Deelder, A., Flad, T., Kostrzewa, M.: Immuno-MALDI-TOF MS: new perspectives for clinical applications of mass spectrometry. Proteomics 9(6), 1442-1450 (2009)
17. Jiang, J., Parker, C.E., Hoadley, K.A., Perou, C.M., Boysen, G., Borchers, C.H.: Development of an immuno-tandem mass spectrometry (iMALDI) assay for EGFR diagnosis. Proteom. Clin. Applications 1 (12), 1651-1659 (2007)

18. Nelson, R.W., McLean, M.A., Hutchens, T.W.: Quantitative determination of proteins by matrix-assisted laser desorption/ionization time-of-flight mass spectrometry. Anal. Chem. 66, 1408-1415 (1994)

19. Wang, J., Sporns, P., Low, N.H.: Analysis of food oligosaccharides using MALDI-MS: quantification of fructooligosaccharides. Journal of Agricultural and Food Chemistry. 47(4), 1549-1557 (1999)

20. Wang, J., Sporns, P.: Analysis of anthocyanins in red wine and fruit juice using MALDI-MS. Journal of Agricultural and Food Chemistry. 47(5), 2009-2015 (1999)

21. Sleno, L., Volmer, D.A.: Toxin screening in phytoplankton: detection and quantitation using MALDI triple quadrupole mass spectrometry. Anal. Chem. 77(5), 1509-1517 (2005)

22. Meng, Z.J., Limbach, P.A.: Quantitation of ribonucleic acids using O18 labeling and mass spectrometry. Anal. Chem. 77(6), 1891-1895 (2005)

23. Yan, W., Gardella, J.A., Wood, T.D.: Quantitative analysis of technical polymer mixtures by matrix assisted laser desorption/ionization time of flight mass spectrometry. J. Am. Soc. Mass Spectrom. 13(8), 914-920 (2002)

24. Chen, H., He, M.: Quantitation of synthetic polymers using an internal standard by matrix-assisted laser desorption/ionization timeof-flight mass spectrometry. J. Am. Soc. Mass Spectrom. 16(1), 100106 (2005)

25. Dean, J.A.: Analytical Chemistry Handbook, p. 4.14. McGraw-Hill, New York (1995)

26. Owens, K.G., Hanton, S.D.: Conventional MALDI Sample Preparation in Li, L. (ed). MALDI Mass Spectrometry for Synthetic Polymer Analysis, John Wiley \& Sons: Hoboken, NJ (2010).

27. Hensel, R.R., King, R.C., Owens, K.G.: Electrospray sample preparation for improved quantitation in matrix-assisted laser desorption/ ionization time-of-flight mass spectrometry. Rapid Commun. Mass Spectrom. 11(16), 1785-1793 (1997)

28. Erb, W.J., Hanton, S.D., Owens, K.G.: A study of gas-phase cationization in matrix-assisted laser desorption/ionization time-offlight mass spectrometry. Rapid Commun. Mass Spectrom. 20(14), 2165-2169 (2006)

29. Martin, K., Spickermann, J., Rader, H.J., Muellen, K.: Why does matrix-assisted laser desorption/ionization time-of-flight mass spectrometry give incorrect results for broad polymer distributions? Rapid Commun. Mass Spectrom. 10(12), 1471-1474 (1996)

30. Wetzel, S.J., Guttman, C.M., Girard, J.E.: The influence of matrix and laser energy on the molecular mass distribution of synthetic polymers obtained by MALDI-TOF-MS. Int. J. Mass Spectrom. 238(3), 215-225 (2004)

31. Guttman, C.M., Flynn, K.M., Wallace, W.E., Kearsley, A.J.: Quantitative mass spectrometry and polydisperse materials: creation of an absolute molecular mass distribution polymer standard. Macromolecules 42, 1695-1702 (2009) 